\title{
Modeling electromechanical properties of ionic polymers
}

\author{
Yu Xiao and Kaushik Bhattacharya \\ California Institute of Technology, 104-44 Caltech, Pasadena, CA 91125, USA
}

\begin{abstract}
We present a multi-scale approach to modeling the electro-mechanical behavior of ionic polymers. We start with a detailed elasto-electro-chemical model which allows for finite deformation. We reduce it to one space dimension appropriate for the commonly used sheet configuration, and demonstrate that steady state solutions display an important boundary layer effect. We conclude with a macroscopic model of a strip of ionic-polymer-metal-composite.
\end{abstract}

\section{INTRODUCTION}

There has been much recent research on the use of electro-active polymers as actuators. ${ }^{1}$ These include the conceptually simplest electrostatic actuators where the compression of an elastomer by oppositely charged electrodes gives the actuation, ionic polymers especially in the form of ionic-polymer-metal composite where the diffusion of ions gives rise to differential deformation which is the basis of actuation, conductive polymers, ferroelectric polymers and even exotic new technologies using carbon nanotubes. Much of the activity has focussed on synthesizing new materials, demonstrating actuation and understanding the electro-chemistry and microscopic mechanism of actuation. These efforts have reached a level of maturity that it is worthwhile to investigate these materials from a more macroscopic point of view with the following objectives.

1. Optimizing the design - the configuration of the polymer, configuration of electrodes etc. - of these actuators to produce desirable force and displacement.

2. Obtaining reduced constitutive models of these actuators, which can be used for designing controllers and for the broader mechanical design of devices.

We have recently presented our efforts to address these questions in electrostatic actuators. ${ }^{2}$ Our current effort is to address these questions in ionic polymers, especially those used in the form of ionic-polymer-metal-composites (IPMC). In doing so, we realized the need to develop a mesoscopic model of such polymers. In these materials, it is impossible to separate the deformation mechanism from the structure, and therefore any optimal design formulation has to start from a suitable mesoscopic model of the polymer.

There has been much activity in understanding the behavior of ionic polymer actuators and also understanding the underlying mechanisms. Nemat-Nasser and $\mathrm{Li}^{3}$ and Nemat-Nasser ${ }^{4}$ have also made much progress in developing a micromechanical model of these polymers. We treat their work as the starting point by starting at the mesoscopic level. We consider a soft elastic medium which allows the diffusion of some ionic species. We regard the elastic medium as insulating - this is appropriate for IPMC, but would have to be revised for conducting polymers. We assume that changes in ionic concentration leads to a local dilatation of the polymer, and model this phenomenologically. Based on this assumptions we develop a model in the setting of finite deformations in Section 2. This model is quasi-static since we further assume that elastic waves and electric pulses are much faster than ionic diffusion. Thus, the only time dependance is through the ionic diffusion. In Section 3, we reduce these equations to one dimension appropriate for a flat sheet with electrodes on the two faces as in IPMC. We show the existence of a pronounced boundary layer. We conclude in Section 4 with a phenomenological model of a strip of IPMC.

We are currently working to find a direct link between the model in Section 3 and the model in Section 4 . We are also working towards evaluating configurations other than flat sheets with the goal of achieving linear (or extensional) actuation. Finally, we plan to expand this framework to conducting and ferroelectric polymers. 


\section{AN ELASTO-ELECTRO-CHEMICAL MODEL OF IONIC POLYMERS}

Consider a ionic polymer occupying a region $\Omega$ in the reference configuration. Suppose it undergoes a deformation $y: \Omega \times(0, T) \rightarrow \mathbb{R}^{3}$. Thus $y(x, t)$ gives the position of the particle $x$ at time $t$. Let $C^{+}(y, t), C^{-}(y, t)$ denote the concentration of cations and anions in the current configuration at time $t$. We assume that the cations are mobile, while the anions are fixed to the back-bone of the polymer. We can then write the total free energy of the system as

$$
\mathcal{F}=\int_{\Omega(t)}\left\{R T C^{+}(y) \ln \frac{C^{+}(y)}{C^{-}(y)}+z F\left(C^{+}(y)-C^{-}(y)\right) \phi(y)-\frac{1}{2} \epsilon\left|\nabla_{y} \phi(y)\right|^{2}\right\} d V_{t}+\int_{\Omega(0)} W_{e}\left(C^{+}(y), \nabla_{x} y\right) d V_{0}
$$

where $T$ is the temperature, $R$ the universal gas constant, $z$ the valency of ions, $F$ the Faraday constant, $\phi$ the electric potential, $\epsilon$ the dielectric constant of vacuum and $W_{e}$ the elastic strain energy which can depend both on cation concentration and on deformation gradient. We give a specific example below.

This expression is not convenient as the first term is an integral over the deformed configuration and the second over the reference configuration. So we change variables to pull back all the terms to the reference configuration. To this end, we define the ionic concentrations in the reference configuration:

$$
C_{0}^{-}(x, t)=C^{-}(y(x, t), t) J(x, t), \quad \tilde{C}^{+}(x, t)=C^{+}(y(x, t), t) J(x, t)
$$

where $J=\operatorname{det} \nabla_{x} y$ is the Jacobian of the deformation gradient. As we assumed, the anions do not diffuse relative to the back-bone of the polymer, i.e. $C_{0}^{-}(x, t)=C_{0}^{-}(x)$. Note that the concentration of anions in the deformed configuration can still change due to the deformation of the polymer. We can now rewrite the total free energy of the system as

$$
\begin{aligned}
& \mathcal{F}=\int_{\Omega(0)}\left\{R T \tilde{C}^{+}(x) \ln \left(\frac{C^{+}(x)}{C_{0}^{-}}\right)+z F\left(\tilde{C}^{+}(x)-C_{0}^{-}\right) \phi(y(x))-\frac{1}{2} \epsilon J(y(x))\left|\nabla_{x} \phi(y(x)) \boldsymbol{F}^{-1}\right|^{2}\right\} d V_{0} \\
&+\int_{\Omega(0)} \widetilde{W}_{e}\left(\tilde{C}^{+}(x), \nabla_{x} y(x)\right) d V_{0}
\end{aligned}
$$

where

$$
\widetilde{W}_{e}\left(\tilde{C}^{+}(x), \nabla_{x} y(x)\right)=W_{e}\left(C^{+}(y(x)), \nabla_{x} y(x)\right) .
$$

and

$$
\boldsymbol{F}=\nabla_{x} y(x)
$$

is the deformation gradient.

As a specific example, let us consider a generalization of the so-called compressible neo-Hookean material. While the exact mechanism of actuation remains under debate, there is a consensus that a change in ionic concentration leads to a dilatation. Thus we introduce a stress-free transformation or 'diffusional stretch' $D\left(\tilde{C}^{+}\right)$. $D$ gives us the preferred stretch if the concentration of cations is $\tilde{C}^{+}$. We now postulate

$$
\widetilde{W}_{e}\left(\tilde{C}^{+}, \boldsymbol{F}\right)=\frac{\lambda}{2} \log ^{2}\left(\frac{J}{D\left(\tilde{C}^{+}\right)^{3}}\right)-\mu \log \left(\frac{J}{D\left(\tilde{C}^{+}\right)^{3}}\right)+\frac{\mu}{2}\left[\frac{\operatorname{tr}\left(\boldsymbol{F}^{T} \boldsymbol{F}\right)}{D\left(\tilde{C}^{+}\right)^{2}}-3\right] .
$$

where $\lambda$ and $\mu$ are lamè-like material constants.

We now use the idea of a 'gradient flow' to derive the governing equations. Carter et al. ${ }^{5}$ provide a detailed discussion of the method and its application to material science. The basic idea is that various quantities - concentration, electric field and deformation - evolve to in such a manner as to reduce the total free energy: in particular it is assumed that the rate of change of these quantities is proportional to the (negative of the) gradient or variation of this energy. This methodology then extends the usual variational derivation of equilibrium equations to the derivation of evolution equations. In order to apply this methodology, one has to take special care about the choice of the function space in which the variation is taken. 
We shall assume that the elastic and electric processes are much faster than the diffusion of cations, and therefore the body is in mechanical and electrical equilibrium at each time while the cation concentration evolves. In other words, we assume that there are no elastic waves or electrodynamical effects in the time-scale of interest. Therefore we choose our variational principle of gradient flow to be

$$
<\tilde{C}_{t}^{+}(x), v>_{H^{-1}, \frac{\tilde{C}+d}{R T}}+\left.\frac{d}{d \varepsilon} \mathcal{F}\left(\tilde{C}^{+}+\varepsilon v, \phi+\varepsilon u, \nabla_{x}(y+\varepsilon w)\right)\right|_{\varepsilon=0}=0
$$

where

$$
<s, t>_{H^{-1}, \frac{\tilde{C}+d}{R T}}=-\int_{\Omega(0)}\left[\left(\nabla_{x} \cdot \frac{\tilde{C}^{+}(x) d}{R T} \nabla_{x}\right)^{-1} s\right] t d V_{0},
$$

$d$ is the diffusion coefficient, and $v, u, w$ are admissible variations of $\tilde{C}^{+}, \phi, y$ respectively.

Expanding Eq. (5) yields the following governing equations (see $\mathrm{Xiao}^{6}$ for details):

$$
\begin{aligned}
& \tilde{C}_{t}^{+}(x)=d \Delta_{x} \tilde{C}^{+}+\frac{d z F}{R T}\left(\nabla_{x} \tilde{C}^{+} \nabla_{x} \phi+\tilde{C}^{+} \Delta_{x} \phi\right)+\frac{d}{R T}\left(\nabla_{x} \tilde{C}^{+} \nabla_{x}\left(\frac{\partial \widetilde{W}_{e}}{\partial \tilde{C}^{+}}\right)+\tilde{C}^{+} \Delta_{x}\left(\frac{\partial \widetilde{W}_{e}}{\partial \tilde{C}^{+}}\right)\right) \text {on } \Omega(0), \\
& \Delta_{y} \phi=-\frac{1}{\epsilon} z F\left(C^{+}(y)-\frac{C_{0}^{-}}{J(y)}\right) \\
& \left(\boldsymbol{\sigma}_{j i}+\boldsymbol{T}_{j i}\right)_{, j}=0 \\
& \text { on } \Omega(t) \text {, } \\
& \text { on } \Omega(t)
\end{aligned}
$$

where

$$
\boldsymbol{\sigma}_{j i}=\frac{1}{J} \frac{\partial \widetilde{W}_{e}}{\partial\left(\nabla_{x} y\right)_{i K}} y_{j, K}
$$

is the Cauchy stress while

$$
\boldsymbol{T}_{j i}=\epsilon \phi_{, i} \phi_{, j}-\frac{1}{2} \epsilon\left|\nabla_{y} \phi\right|^{2} \delta_{j i}
$$

is the Maxwell electrostatic stress.

The first of these equations, Eq. (7), is the diffusion equation for the cations. Note that the first two terms on right hand side correspond exactly to the classical Einstein-Nernst diffusion equation. The third term is the effect of elasticity: it says that a gradient in the strain energy can also affect the flux of cations. The second of these equations, Eq. (8), is the classical Maxwell's equations adopted to electrostatics. The third is the equation of mechanical equilibrium. Note that the stress has two contributions, the Cauchy stress from the deformation gradient and the Maxwell stress from the electrostatics. Note that this equilibrium equation is also coupled to the cation concentration since the strain energy depends on concentration.

For the specific example of elastic energy density given in Eq. (4), the Cauchy stress

$$
\boldsymbol{\sigma}_{j i}=\frac{1}{\operatorname{det} \boldsymbol{F}}\left[\lambda \log \left(\frac{\operatorname{det} \boldsymbol{F}}{D\left(\tilde{C}^{+}\right)^{3}}\right)-\mu\right] \delta_{j i}+\frac{\mu}{(\operatorname{det} \boldsymbol{F}) D\left(\tilde{C}^{+}\right)^{2}}\left(\boldsymbol{F} \boldsymbol{F}^{T}\right)_{j i}
$$

depends on both concentration and deformation gradient, while the contribution of elasticity to the diffusion potential is

$$
\frac{\partial \widetilde{W}_{e}}{\partial \tilde{C}^{+}}=\left[-3 \lambda \log \left(\frac{\operatorname{det} \boldsymbol{F}}{D\left(\tilde{C}^{+}\right)^{3}}\right)+3 \mu-\mu \frac{\operatorname{tr} \boldsymbol{F}^{T} \boldsymbol{F}}{D\left(\tilde{C}^{+}\right)^{2}}\right] \frac{D^{\prime}\left(\tilde{C}^{+}\right)}{D\left(\tilde{C}^{+}\right)}
$$

In summary, the behavior of an ionic polymer subject to cation diffusion is given by three coupled non-linear partial differential equations, which have to be supplemented with appropriate boundary and initial conditions. We note finally, that these equations have to be solved in the deformed configuration, which is unknown apriori. One can either pull them back to the reference configuration which introduces further complications or solve them incrementally.

Addressing these issues is beyond the scope of this short note, and the reader is referred to Xiao ${ }^{6}$ for details. 


\section{SIMPLIFIED ONE-DIMENSIONAL MODEL}

Ionic polymers are often fabricated and used in a thin-sheet configuration. Ionic-polymer-metal-composite (IPMC) is a typical example. Here, a thin sheet of this polymer is coated with metal electrodes on both sides and a voltage difference is applied between them. In this situation it is natural to expect that the diffusion to be one-dimensional (in the thickness direction), and cation concentration, electric potential and strain to vary only along the thickness. We can therefore reduce these equations to one space dimension: this is a nontrivial task in the setting of finite deformations and the reader is referred to $\mathrm{Xiao}^{6}$ for details. It turns out that the main nonlinearity occurs in the electro-chemistry, and as a means of understanding this we also ignore the coupling between the electro-chemistry and the elasticity in this short note. Under these assumptions equations Eqs. (7), (8) reduce to

$$
\begin{gathered}
C_{t}^{+}=d C_{x x}^{+}+d \frac{z F}{R T} C_{x}^{+} \phi_{x}-d \frac{z^{2} F^{2}}{\epsilon R T}\left(C^{+}-C^{-}\right) C^{+}, \\
\phi_{x x}=-\frac{z F}{\epsilon}\left(C^{+}-C^{-}\right)
\end{gathered}
$$

with boundary conditions

$$
\begin{aligned}
& J(0, t)=0, \quad \phi(0, t)=0, \\
& J(L, t)=0, \quad \phi(L, t)=\phi_{0}
\end{aligned}
$$

and initial condition

$$
C^{+}(x, 0)=C^{-}=\text {constant }
$$

where $J=-d\left(C_{x}^{+}+\frac{z F C^{+}}{R T} \phi_{x}\right)$. The boundary conditions Eq. (16) state that there is no ionic flux at the two faces, and that the voltage is precribed at the two faces. We point out that these equations are essentially those considered by other authors including Nemat-nasser and $\mathrm{Li},{ }^{3}$ except we retain the last nonlinear term. We shall see that this term is crucial as it determines the boundary layer.

It is convenient to write these equations in non-dimensional form. Let

$$
z=\frac{x}{L}, \quad \tau=d \frac{z^{2} F^{2} C-}{\epsilon R T} t, \quad u=\frac{C^{+}}{C^{-}}, \quad \psi=\frac{\phi}{\phi_{0}}
$$

and

$$
\alpha=\frac{z F \phi_{0}}{R T}, \quad \varepsilon=\frac{\epsilon R T}{L^{2} z^{2} F^{2} C^{-}} .
$$

We can then rewrite the governing equations, Eq. (14) to Eq. (17), as

$$
\begin{gathered}
u_{\tau}=\varepsilon\left(u_{z z}+\alpha u_{z} \psi_{x}\right)+(1-u) u, \\
\varepsilon \alpha \psi_{z z}=1-u
\end{gathered}
$$

with boundary conditions

$$
\begin{gathered}
\psi(0, \tau)=0, \quad \psi(1, \tau)=1 \\
u_{z}(0, \tau)+\alpha u \psi_{z}(0, \tau)=u_{z}(1, \tau)+\alpha u \psi_{z}(1, \tau)=0
\end{gathered}
$$

and intitial condition

$$
u(z, 0)=1
$$

In a typical material, $\varepsilon=10^{-15}$ and hence we can regard it as a small parameter. Therefore we expect that a boundary layer comparable in thickness to $\sqrt{\varepsilon}$ in non-dimensional length (or equivalently comparable in thickness to $\sqrt{\frac{\epsilon R T}{z^{2} F^{2} C^{-}}}$which is of the order of Angstoms in physical length). This thickness appears to be smaller than those estimated experimentally recently by Nemat-Nasser. ${ }^{4}$ We believe that there are two reasons. First, the estimate here ignores the effect of elasticity. Recall from Eq. (7) that gradients in the strain energy affect the cation concentration. This would resist the formation of sharp gradients in concentration and spread out the boundary layer. Second, IPMC is a graded material so that we do not have sharp transition from metal to polymer. Instead the metal penetrates the polymer and our treatment of this layer as strictly insulating is incorrect. This would affect the boundary layer.

We now look at the steady state equations to understand this boundary layer. 

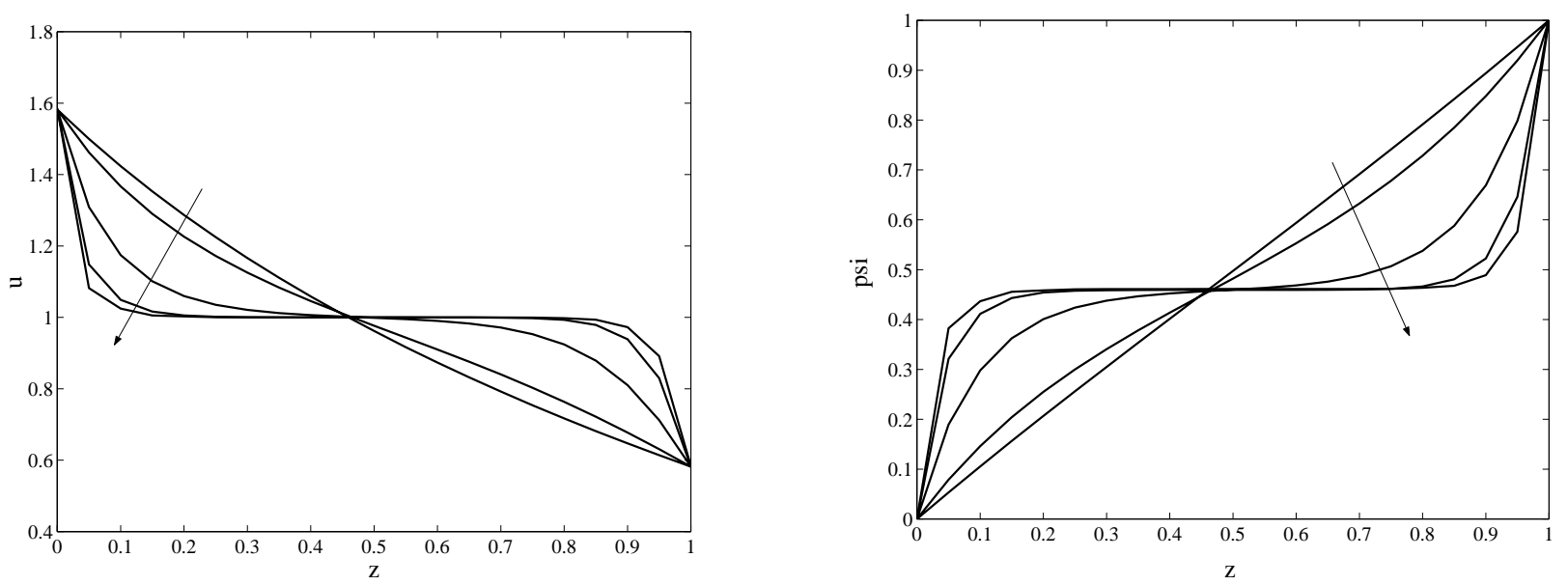

Figure 1. Distribution of cations in steady state (left) and the corresponding normalized electric potential (right). In these plots $\alpha=1$ and $\varepsilon$ varies from 1 to 0.0001 . Direction of arrow indicates the decrease of $\varepsilon$

\subsection{Steady state solutions}

In steady state, the time $(\tau)$ derivative in Eq. (20) is zero, and one obtains significant simplifications. In fact, the change of dependent variable

$$
w=\log u,
$$

along with some manipulations (see $\mathrm{Xiao}^{6}$ for details) allow us to decouple the equations of concentration and electric field. We thus have to solve only a single ordinary differential equation with some unusual boundary conditions:

$$
\begin{gathered}
w_{z z}=\frac{1}{\varepsilon}\left(e^{w}-1\right) \quad \text { on }(0,1), \\
w(0)=w(1)+\alpha \\
w_{z}(0)=w_{z}(1)
\end{gathered}
$$

for the (logarithm of the) concentration, and then obtain the electric potential explicitly

$$
\psi(z)=\frac{1}{\alpha}(w(0)-w(z))
$$

We have developed a technique to solve Eq. (25) to Eq. (27). We use a phase plane analysis to first determine the value $w(0)$, and then integrate explicitly. Figures 1 and 2 show the behavior of the non-dimensional concentration $u$ and electrostatic potential $\psi$ as a function of non-dimensional position $z$ along the thickness of the sheet. In Figure 1 , we hold the non-dimensional parameter $\alpha \operatorname{fixed}(\alpha=1)$ while we vary $\varepsilon$ from 1 to 0.0001 . The direction of the arrow shows decreasing $\varepsilon$. Though we estimate $\varepsilon$ to be order $10^{-15}$, we show the results only for larger values for ease of visualization. In Figure 2, we hold the non-dimensional parameter $\varepsilon$ fixed $(\varepsilon=1)$ while we vary $\alpha$ from 0.5 to 2.0 .

Notice the pronounced boundary layer that forms when $\varepsilon$ becomes small, and also notice that the boundary layer becomes thinner as the $\varepsilon$ becomes smaller. There is a small layer of accumulation and depletion of cations near negative and positive electrodes respectively. Further, most of the electric potential is dropped in the boundary layer. This clearly shows that most of the actuation arises very close to the electrodes, and the central region does not contribute much to the behavior.

\section{A REDUCED MODEL OF MACROSCOPIC BEHAVIOR}

Consider a thin sheet of ionic polymer coated with electrodes on the two surfaces (like IPMC), and suppose a voltage difference is applied across the two electrodes. This causes a change in the cation concentration as we saw in the 

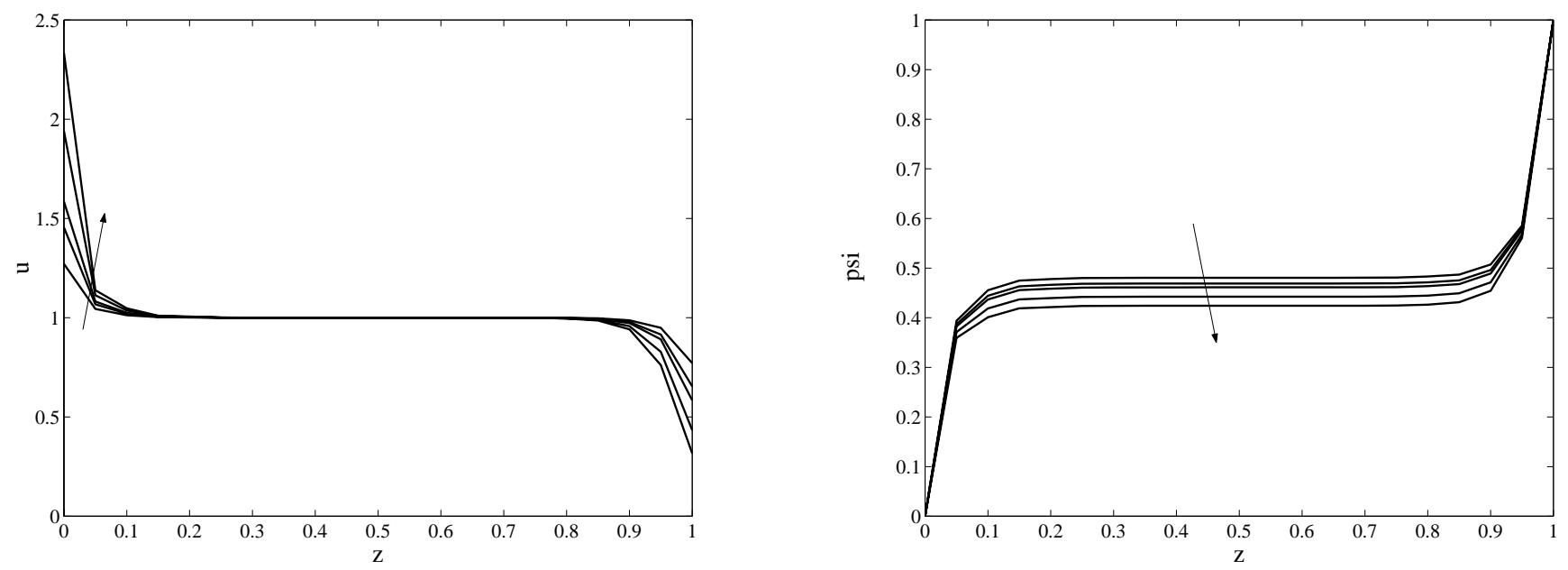

Figure 2. Distribution of cations in steady state (left) the correspoding normalized electric potential (right).In these plots $\varepsilon=0.0001$ and $\alpha$ varies from 0.5 to 2.0. Direction of arrows indicates the increase of $\alpha$

previous section. This in turn leads to a deformation of the sheet. However the deformation is not uniform, but varies across the thickness along with the cation concentration $\tilde{C}^{+}$. Thus one face of the sheet stretches, while the other shrinks giving rise to a net curvature in the sheet. We call this curvature - the curvature induced by the electric field in the absence of any loading - the eigen-curvature. This eigen-curvature would in general be bi-axial as the polymer is isotropic in the plane of the sheet. Thus we would expect the deformation of the polymer sheet to involve bending in two directions. However, there is an important issue that one has to consider.

To understand this, consider a flat sheet of paper. It is very easy to roll it up into cylinders and cones, where the bending is only along one axis at any point. On the other hand it is very difficult to give it a biaxial curvature. This is easily appreciated by trying to drape this piece of paper over a sphere; it resists by forming wrinkles and folds. The reason is that biaxial curvature requires us to stretch the sheet, while uniaxial curvature does not require this stretching. Further, stretching requires much larger forces, equivalently it is much more energetically expensive. Thus the sheet resists stetching by bifurcating into the wrinkled and folded shape.

Let us now return to our ionic polymer sheet which is initially flat. As a voltage is applied to the electrode, and the eigen-curvature increases, the sheet initially deforms biaxially. However as the eigen-curvature increases beyond a critical value, the stretching induced by the biaxial curvature becomes energetically too expensive and the sheet 'bifurcates' into a uniaxially deformed state. Thus, the deformation of the sheet can be uniaxial even through the eigen-curvature is biaxial. This bifurcation has recently been discussed extensively by Freund ${ }^{7}$ in the context of thin films on silicon wafers where thermal and epitaxial mismatch gives rise to eigen-curvature.

We are interested in the deformation of a narrow strip of IMPC (for example, $0.17 \mathrm{~mm} \times 2 \mathrm{~mm} \times 20 \mathrm{~mm}$ ). Suppose one end of the strip is clamped while the other end is free and a voltage is applied across the thickness. This narrow strip bifurcates very quickly into a uniaxial bending, much like a beam. Since there is no load at the free end, and since we assume good electrodes, the curvature of the beam would be uniform. Now suppose we apply a load $F$ to the free end. The curvature would no longer be uniform and we are interested in finding this. We now discuss a macroscopic theory to capture this. This is a nonlinear Euler-Bernouli beam model, augumented for eigen-curvature.

Consider an IPMC strip shown in the Figure 3, and assume for now that the applied force $F$ is zero. The strip bends in response to an applied electric field, and as discussed the curvature is uniform and equal to the eigencurvature which we denote as $\kappa$. If we apply a step voltage to the beam, this curvature evolves with time as shown in Figure $3,^{8}$ and we have to solve the Eq. (14) to Eq. (17) to find the evolution of this eigen-curvature. This however is rather difficult, and we proceed phenomenologically. We notice that we can fit the experimental observations to an exponential profile. Therefore we assume that the evolution of the eigen-curvature with time is decribed by

$$
\kappa(t)=\kappa_{v} V-\left(\kappa_{v} V-\kappa_{0}\right) \exp \left(-\frac{t}{\tau}\right)
$$



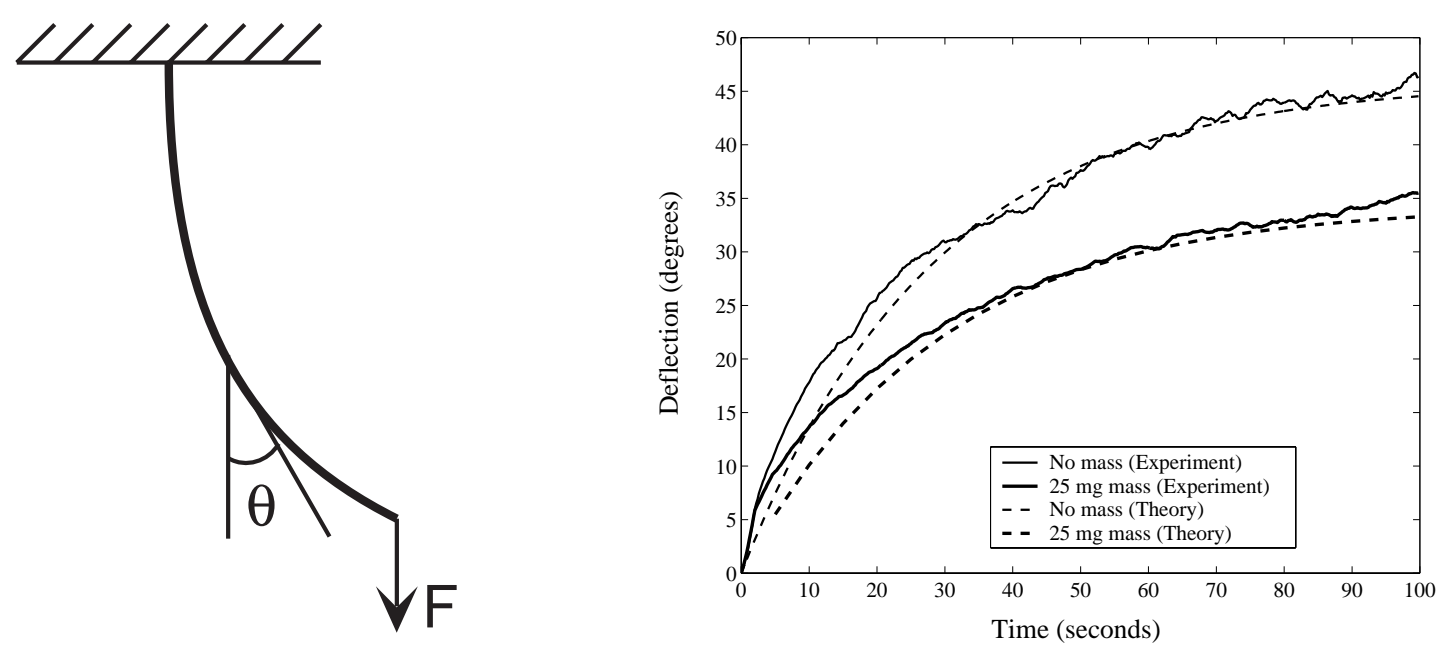

Figure 3. A typical configuration of a IPMC strip or beam clamped at one end and subjected to a load $F$ at the other (left). The response of the beam in terms of the deflected angle of the tip to a step voltage across the thickness (right).

where $\kappa_{v}$ denotes the saturation curvature per unit applied voltage, $\tau$ is the response time constant (these are both regarded as material constants), and $\kappa_{0}$ is the initial curvature. For a more general time dependant applied voltage, the response can be described by a first order ordinary differential equation

$$
\frac{d \kappa}{d t}=\frac{1}{\tau}\left(\kappa_{v} V-\kappa\right)
$$

We emphasize that this equation is phenomenological, and we plan to use the unsteady model of Section 3 to obtain it directly in the future.

Let us now consider more general deformation of the beam shown in Figure 3. Assuming the beam is inextensible, we can describe the deformation of the beam using a function $\theta(s)$ where $\theta$ is the angle shown in Figure 3 and $s$ is the arc-length along the beam. For given eigen-curvature $\kappa$, the (excess) strain energy of the beam is

$$
W=\int_{0}^{l} \frac{1}{2} E I\left(\theta^{\prime}-\kappa\right)^{2} d s
$$

where' denotes differentiation with respect to $s$, and $E I$ is the flexural rigidity. For an applied force $F$ as shown in Figure 3, the total potential energy of the beam is

$$
\begin{aligned}
L & =W-\text { work done by external force } \\
& =\int_{0}^{l}\left(\frac{1}{2} E I\left(\theta^{\prime}-\kappa\right)^{2}+F(1-\cos \theta)\right) d s .
\end{aligned}
$$

From the principle of the virtual work $\delta L=0$, we obtain the equilibrium equation and boundary conditions

$$
\begin{gathered}
E I \theta^{\prime \prime}-F \sin \theta=0, \\
\theta(0)=0, \quad \theta^{\prime}(l)=\kappa .
\end{gathered}
$$

Eq. (33) is a simple second order semi-linear ordinary differential equation with two-point boundary conditions, we can solve it semi-analytically for a given $\kappa$.

This completes the simple phenomenological model which has three material constants $E$, $\kappa_{v}$, and $\tau$, which respectively are the (effective) elastic modulus, the saturation curvature at unit applied voltage and the time-constant.

The solution procedure for a fixed applied load $F$ and given time-dependant $V(t)$ is as follows. At each time $t$, we integrate Eq.(30) to obtain the eigen-curvature $\kappa$. For this eigen-curvature we solve Eq.(33) to obtain the deformation $\theta(s)$. We repeat for each time $t$. 

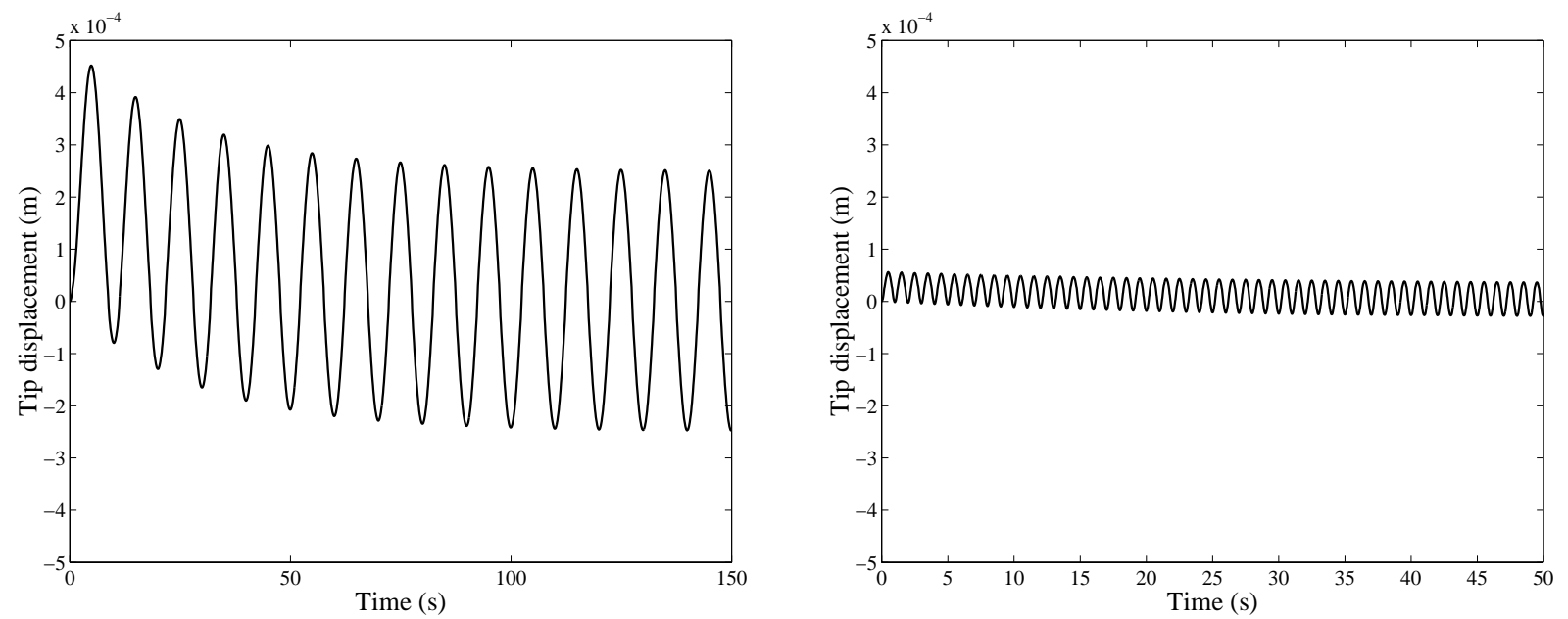

Figure 4. Response of the beam in terms of tip displacement subjected to a sinusoidal voltage across the thickness with tip mass $20 \mathrm{mg}, V_{0}=1 \mathrm{~V}$ (left: frequency $=0.1 \mathrm{~Hz}$, right: frequency $=1.0 \mathrm{~Hz}$ )

By applying different time-dependant voltages and different forces and fitting them to the model above, we can evaluate all the material constants $E, \kappa_{v}, \tau$. Our preliminary investigation to experiments of Bar-Cohen and Leary ${ }^{8}$ shows

$$
E=20 \mathrm{MPa}, \quad \kappa_{v}=0.07 \mathrm{~V}^{-1} \mathrm{~mm}^{-1}, \quad \tau=51 \mathrm{~s}
$$

This model can be easily adopted to many complicated loadings and applied voltages. We conclude with a simple example to demonstrate this point. Suppose we start at an initial straight configuration and apply a sinusoidal voltage

$$
V=V_{0} \sin \omega t
$$

Then the response of the model is shown in Figure 4 for two different frequencies $\omega$. Note that one sees fairly large response at small frequencies, but virtually no response at higher frequencies.

Acknowledgement. It is our pleasure to acknowledge many interesting and stimulating discussions with Virginia Olazábal and José-Maria Sansiñena, and the partial financial support of the Defense Advanced Research Projects Agency and the Caltech President's Fund.

\section{REFERENCES}

1. Y. Bar-Cohen, Electroactive polymer (EAP) actuators as artifical muscles. Reality and science fiction, SPIE, to appear, 2000.

2. K. Bhattacharya, J.Y. Li and Y. Xiao, Electromechanical models for optimal design and effective behavior of electroactive polymers, in Electroactive polymer (EAP) actuators as artifical muscles. Reality and science fiction (ed. Y. Bar-Cohen), SPIE to appear, 2000.

3. S. Nemat-Nasser and J.Y. Li, Electromechanical response of ionic polymer-metal composites, Journal of Applied Physics, v. 87, no. 7, 2000, p.3321-3331.

4. S. Nemat-Nasser, C.W. Thomas, Ionomeric Polymer-Metal Composites, in Electroactive polymer (EAP) actuators as artifical muscles. Reality and science fiction (ed. Y. Bar-Cohen), SPIE to appear, 2000.

5. W.C. Carter, J.E. Taylor, J.W. Cahn, Variational methods for microstructural-evolution theories, JOM, (49)1997, p.30-36.

6. Y. Xiao, A multiscale modelling framework for electro-active polymers, Ph.D. Thesis, California Institute of Technology, forthcoming. 
7. L.B. Freund, Substrate curvature due to thin film mismatch strain in the nonlinear deformation range, Journal of the Mechanics and Physics of Solid, (48)2000, p.1159-1174.

8. Y. Bar-Cohen and S. Leary, Electroactive Polymers(EAP) Characterization Methods, Smart Structures and Materials 2000: Electroactive Polymer Actuators and Devices (EAPAD), Proceedings of SPIE's 7th Annual International Symposium on Smart, Structures and Materials, Newport Beach, CA, 5-9 March, 2000, p39874004 . 